\title{
Retraction Note to: Discovering Graphical Visual Features for Abnormal Semantic Event Detection
}

\section{Fenghua Wang ${ }^{1} \cdot$ Yuhui $\mathrm{Ma}^{1} \cdot$ Yanjuan $\mathrm{Jin}^{2} \cdot$ Ying Jiang $^{1} \cdot$ Yunye Wang ${ }^{1}$}

Published online: 10 June 2019

(C) Springer Science+Business Media, LLC, part of Springer Nature 2019

Retraction Note to: Multimed Tools Appl (2018) 77:3245-3260.

https://doi.org/10.1007/s11042-017-5057-3

The Editor-in-Chief has retracted this article [3], which was published as part of special issue "Semantic Concept Discovery in MMData", because the article shows substantial text overlap most notably with the articles cited $[1,2,4,5]$.

The authors have not responded to correspondence about this retraction.

The online version of the original article can be found at https://doi.org/10.1007/s11042-017-5057-3.

Fenghua Wang

wang_fenghua@zj.sgcc.com

Yuhui Ma

ma_yuhui@zj.sgcc.com

Yanjuan Jin

Jin_yanjuan@zj.sgcc.com

Ying Jiang

Jiang_ying@zj.sgcc.com

Yunye Wang

wang_yunye@zj.sgcc.com

1 State Grid Zhejiang Electric Power Company Information \& Telecommunication Branch, Hangzhou, China

2 Hangzhou Dayou Science and Technology Development Co., Ltd., Hangzhou, China 


\section{References}

1. Chang X, Ma Z, Lin M et al (2017) Feature interaction augmented sparse learning for fast Kinect motion detection. IEEE Trans Image Process. https://doi.org/10.1109/TIP.2017.2708506

2. Nie X, He D, Chan S et al (2016) Network anomaly detection using unsupervised feature selection and density peak clustering. Lect Notes Comput Sci. https://doi.org/10.1007/978-3-319-39555-5_12

3. Wang F, Ma Y, Jin Y et al (2018) Discovering graphical visual features for abnormal semantic event detection. Multimed Tools Appl 77:3245. https://doi.org/10.1007/s11042-017-5057-3

4. Zhang L, Song M, Li N et al (2009) Feature selection for fast speech emotion recognition. In: Proceedings of the 17th ACM International Conference on Multimedia. https://doi.org/10.1145/1631272.1631405

5. Zhang L, Tao D, Liu X et al (2013) Grassmann multimodal implicit feature selection. Multimedia Systems. https://doi.org/10.1007/s00530-013-0317-1

Publisher's note Springer Nature remains neutral with regard to jurisdictional claims in published maps and institutional affiliations. 